\title{
Otic Langerhans Cell Histiocytosis in Adults: A Report of Two Cases
}

\author{
Nasser Almutairia, b, Jihad Almahdi ${ }^{\mathrm{a}}$, Mohammed Halawani ${ }^{\mathrm{a}}$
}

\begin{abstract}
We presented two cases of Langerhans cell histiocytosis (LCH) in which the temporal bone and hearing center in the brain stem were affected. We discuss the diagnosis and management of this disease and how it presents if the temporal bone is involved. The first case involved a patient with destructive lesions in the temporal bone, who responded to medical management, while the second case involved a patient with brain stem lesions, who did not improve on chemotherapy. The findings from these two cases suggest that a differential diagnosis of $\mathrm{LCH}$ should be considered in patients with persistent ear discharge or hearing loss that does not respond to conventional antibiotic treatment, and that imaging studies are required for any atypical presentation.
\end{abstract}

Keywords: Otic Langerhans; Histiocytosis; Hearing loss; Ear discharge

\section{Introduction}

Langerhans cell histiocytosis (LCH) generally affects the pediatric population. However, in 1979, McCaffrey et al [1] reported that LCH exhibits a secondary peak incidence in individuals between 30 and 39 years of age. According to a report from the International Registry of the Histiocyte Society, published by Arico et al in 2003, the mean age of adults with LCH is 35 years [2].

Ear and temporal bone involvement occurs in $14-61 \%$ of children with LCH; however, it is very rare in adults [3]. Ear involvement in $\mathrm{LCH}$ can present as chronic otitis externa or otitis media, an external ear canal mass or polyp, post-auricular swelling, conductive hearing loss, vertigo or, rarely, facial paralysis [3]. Furthermore, it is bilateral in $30 \%$ of patients, and is more likely to occur as part of multifocal or systemic LCH [3]. However, up to $25 \%$ of patients present only with

Manuscript submitted February 12, 2017, accepted February 23, 2018

aDepartment of ORL, National Guard Hospital, PO Box 71751, Riyadh, Saudi Arabia

${ }^{b}$ Corresponding Author: Nasser Almutairi, Department of ORL, National Guard Hospital, PO Box 71751, Riyadh, Saudi Arabia.

Email: Dr.nasser-20@hotmail.com

doi: https://doi.org/10.14740/jmc2917w symptoms of ear involvement [4].

Here, we describe the clinical course and treatment of LCH with ear involvement in a 25 -year-old man and a 38-yearold woman. These individuals presented with signs and symptoms that can easily be missed. Even though LCH is a systematic disease, and rarely affects the hearing organs, the man presented with ear symptoms only, while the woman exhibited ear symptoms in addition to vocal cords paralysis. Case files were obtained and reviewed with ethics approval from each hospital research center.

\section{Case Reports}

\section{Case 1}

Case 1 involved a 25-year-old male soldier with no relevant medical history who presented with a chief complaint of recurrent discharge from the right ear for the previous 2 years. Treatment with antibiotics was ineffective, and he developed progressive hearing loss. There was no history of ear pain, fever, tinnitus, vertigo, headache, ear fullness, trauma, or previous ear surgery. Furthermore, there was no history of neurological or constitutional symptoms. On physical examination, the right ear exhibited an unhealthy and fragile epithelium in the external auditory canal (EAC), with minimal, yellowish discharge. The tympanic membrane was intact, with no keratin tissue or masses. Examinations of the facial nerve and other cranial nerves revealed no abnormalities. Findings from examinations of the left ear were unremarkable. Audiological assessments indicated fluid accumulation in the right middle ear accompanied by profound sensorineural hearing loss (SNHL). Function in the left ear was normal. Computed tomography (CT) of the temporal bone revealed destruction of the right mastoid process, squamous temporal bone, greater wing of the sphenoid bone, temporal fossa, EAC, and lateral and medial walls of the middle ear, with erosion of the lateral semicircular canal, apical turn of the cochlea, and tegmen tympani. A welldefined lytic lesion was also observed in the left occipital bone. Subsequent soft tissue analysis using magnetic resonance imaging (MRI) revealed fluid accumulation with enhancement in the right mastoid air cells, and middle and inner ear, up to the EAC. Abnormal enhancement in the dura of the middle and posterior fossae was also observed. Fluid attenuation inversion recovery images revealed an isointense, well-defined lesion that grossly measured $2.2 \times 1.2 \mathrm{~cm}$ in the left occipital bone, 
with no defined post-contrast enhancement.

The patient was transferred to the operating room for the insertion of a myringotomy tube in the right ear. In addition, a team of neurosurgeons performed a biopsy of the occipital lesion. Histopathological analysis of the specimen led to a final diagnosis of LCH. The patient was treated by both ENT and neurosurgery specialists, and the discharge disappeared after a trial of ofloxacin ear drops administered for 3 weeks. Subsequently, he was referred to a hemato-oncology team and was administered systemic steroids with vincristine chemotherapy. After a discussion with the hemato-oncology team, it was decided that this patient would benefit more from radiation therapy than from surgery; surgery was ruled out because of the diffuse temporal bone destruction.

Currently, the patient is free from symptoms and is being followed up in the Hematology Department. Although he is currently in regular follow-up, he will undergo radiation therapy if symptoms recur.

\section{Case 2}

Case 2 involved a 38-year-old woman with a known history of bronchial asthma and allergic rhinitis, who referred to ENT with bilateral vocal cord paralysis. A few years previously, she had developed acute otitis media and facial asymmetry that was successfully treated with antibiotics and steroids. In the same year, she developed progressive swelling in the left parietal region. Subsequent imaging studies revealed a lytic lesion in the parietal region and small nodular lesions in the regions of the hypothalamus and brain stem. She was evaluated by a multidisciplinary team consisting of endocrinologists, hematologists, orthopedic specialists, neurosurgeons, and neurologists.

She exhibited radiological signs of improvement after steroid therapy. At that time, multiple biopsy specimens were obtained from the sinus, skull lesion, and bone marrow, which suggested inflammatory changes with insignificant results. Cerebrospinal fluid examination yielded non-specific findings, with no signs of malignancy. She subsequently developed knee pain, and a knee biopsy revealed LCH.

A team of hematologists initiated steroid treatment as well as chemotherapy; however, the disease progressed to the vagus nerve nucleus in the brain stem, which led to bilateral vocal cord paralysis. A tracheostomy was performed and audiological assessment revealed moderate bilateral SNHL. CT and MRI revealed a lytic lesion in the left parietal bone and nodular lesions in the brain stem. It was speculated that the lesions compressing the brain stem were the reason for the hearing loss and vocal cord paralysis. The patient is currently in stable condition, with no improvement, and remains on chemotherapy.

\section{Discussion}

We described two cases of otic LCH in adults. $\mathrm{LCH}$ is a disorder that primarily affects children, but also occurs in adults of all ages. Affected individuals produce excessive Langerhans cells or histiocytes, a type of white blood cell that protects the body from infection in healthy individuals. In LCH, these cells multiply in large numbers and accumulate in certain areas of the body, which leads to the formation of tumors known as granulomas. The symptoms of LCH vary among affected individuals, depending on the extent of the disease and the organs involved. In most cases, however, this condition is not life threatening, although some patients experience life-long problems associated with the disease [5].

The disease can affect virtually every organ, including the skin, bones, lymph nodes, bone marrow, liver, spleen, lungs, gastrointestinal tract, thymus, central nervous system, and hormone glands, of which the skin, bone, lymph nodes, nervous system, and liver are most commonly involved [6]. The symptoms may range from localized bone lesions or skin disease, to multiple organ involvement and severe dysfunction [6].

The exact cause of $\mathrm{LCH}$ remains unknown [7, 8]. It may be triggered by an unusual reaction of the immune system to substances commonly found in the environment. It is not considered to be an infection or cancer, and is not hereditary or contagious [7].

Tests for LCH include bronchoscopy with biopsy, X-ray, skin biopsy, bone marrow biopsy, complete blood count, and pulmonary function tests. Since LCH is sometimes associated with cancer, $\mathrm{CT}$ and biopsy may be required to rule out malignancy $[6,9]$.

Treatment for $\mathrm{LCH}$ depends on the individual, and may differ depending on the type and severity of the condition, as well as the organs and/or systems involved. In some cases, the disease spontaneously resolves, in others, limited surgery and small doses of radiation therapy or chemotherapy are required, depending on the extent of the disease. Treatment is planned after complete evaluation of the patient, with the primary aim of minimum intervention to keep the disease under control [5]. Localized disease may be treated with surgical excision and intralesional steroids [10-13], whereas a more conservative approach is advocated for systemic disease. Immunosuppression has been used to treat systemic LCH; however, knowledge of its efficacy is limited by the small number of studies in which this modality has been used [11]. The mainstay of treatment for systemic disease is chemotherapy, with vinblastine commonly used in combination with a steroid [11].

The prognosis (i.e., chance for recovery) of $\mathrm{LCH}$ varies greatly among patients. In the majority of children, the disease resolves. The prognosis is primarily dependent on the number of organ systems involved, the severity of organ involvement and, to a lesser extent, the age at which symptoms develop [6, 9]. In general, young patients and patients with disseminated disease and organ dysfunction tend to have a poorer prognosis $[6,9]$. Newborns who present only with skin lesions tend to do well. Therefore, the age at presentation is only important when multiple organs are affected. Patients with liver, spleen, lung, and/or bone marrow involvement generally have a poor prognosis. In a multicenter study, it was shown that the best prognostic indicator was the patient's response to chemotherapy during the first 6 weeks. Accordingly, more aggressive treatment is recommended for patients who do not respond positively within the first 6 weeks of treatment [9].

The findings from case 1 demonstrate that persistent ear 
discharge in adults may be associated with $\mathrm{LCH}$; this is a rare presentation in the adult population and can be missed easily [3]. When patients with persistent ear discharge present with radiological evidence of a soft tissue mass within the middle ear and EAC, with or without bony erosion and other skull lesions, LCH should be considered in the differential diagnosis. CT is useful for delineating bony involvement and reveals bony destruction of the petrous apex, while MRI provides information about the extent of any soft tissue involvement [14]. Case 2 exhibited hearing loss that was probably the result of central nervous system involvement, although this is rarely mentioned in the literature. The patient exhibited vocal cord paralysis; unfortunately, her disease was disseminated. Sometimes the biopsy is not giving clear picture of the disease as this case underwent multiple biopsies from multiple sites and were not diagnostic. She is currently in a stable condition and is undergoing chemotherapy; her hearing level has remained virtually the same.

We do not recommend surgery for the management of otic $\mathrm{LCH}$, because of extreme difficulty in achieving total resection of the diseased tissue. Furthermore, surgery is associated with the risk for serious complications, such as conductive hearing loss and SNHL, facial nerve paralysis, postoperative fistula formation, and persistent disease activity [13].

\section{Conclusions}

The findings from these two cases suggest that a differential diagnosis of LCH should be considered in patients with persistent ear discharge, not responding to conventional antibiotic treatment, and those with soft tissue lesions involving the temporal bone or hearing centers in the brain stem. Moreover, physicians should be aware that this disease can also manifest as hearing loss alone, caused by central or peripheral system involvement, and imaging is requisite for any cases with atypical presentation.

\section{References}

1. McCaffrey TV, McDonald TJ. Histiocytosis X of the ear and temporal bone: review of 22 cases. Laryngoscope. 1979;89(11):1735-1742.

2. Arico M, Girschikofsky M, Genereau T, Klersy C, McClain K, Grois N, Emile JF, et al. Langerhans cell histiocytosis in adults. Report from the International Registry of the Histiocyte Society. Eur J Cancer. 2003;39(16):23412348.

3. Whitaker EG, Cerenko D, Muller S, Hudgins P. Multifocal langerhans' cell histiocytosis involving bilateral temporal bones, lungs, and hypothalamus in an adult. Skull Base Surg. 1999;9(1):51-56.

4. Quesada P, Navarrete ML, Perello E. Eosinophilic granuloma of the temporal bone. Eur Arch Otorhinolaryngol. 1990;247(3):194-196.

5. LCH in Children. Histiocytosis Association of America. $<$ www.histio.org $>$ Accessed February 2, 2017.

6. Grifo AH. Langerhans cell histiocytosis in children. J Pediatr Oncol Nurs. 2009;26(1):41-47.

7. $\mathrm{LCH}$ in Children. Histiocytosis Association of America. $<$ www.histio.org $>$ Accessed February 18, 2010.

8. Shea CR, Boos MD. Langerhans cell histiocytosis. Medscape. 2016.

9. Satter EK, High WA. Langerhans cell histiocytosis: a review of the current recommendations of the Histiocyte Society. Pediatr Dermatol. 2008;25(3):291-295.

10. Saliba I, Sidani K, El Fata F, Arcand P, Quintal MC, Abela A. Langerhans' cell histiocytosis of the temporal bone in children. Int J Pediatr Otorhinolaryngol. 2008;72(6):775786.

11. Donadieu J, Chalard F, Jeziorski E. Medical management of langerhans cell histiocytosis from diagnosis to treatment. Expert Opin Pharmacother. 2012;13(9):1309-1322.

12. Nicollas R, Rome A, Belaich H, Roman S, Volk M, Gentet JC, Michel G, et al. Head and neck manifestation and prognosis of Langerhans' cell histiocytosis in children. Int J Pediatr Otorhinolaryngol. 2010;74(6):669-673.

13. Irving RM, Broadbent V, Jones NS. Langerhans' cell histiocytosis in childhood: management of head and neck manifestations. Laryngoscope. 1994;104(1 Pt 1):64-70.

14. Marioni G, De Filippis C, Stramare R, Carli M, Staffieri A. Langerhans' cell histiocytosis: temporal bone involvement. J Laryngol Otol. 2001;115(10):839-841. 